\title{
BASAL NITRIC OXIDE EXPRESSES ENDOGENOUS CARDIOPROTECTION DURING REPERFUSION BY INHIBITION OF NEUTROPHIL-MEDIATED DAMAGE AFTER SURGICAL REVASCULARIZATION
}

\author{
Hiroki Sato, $\mathrm{MD}^{\mathrm{a}}$ \\ Zhi-Oing Zhao, $\mathrm{MD}, \mathrm{PhD}^{\mathrm{a}}$ \\ James E. Jordan, BS ${ }^{\mathrm{b}}$ \\ James C. Todd, $\mathrm{MD}^{\mathrm{a}}$ \\ Robert D. Riley, MD $^{\mathrm{a}}$ \\ C. Spencer Taft, $\mathrm{BS}^{\mathrm{a}}$ \\ John W. Hammon, Jr., MD ${ }^{\mathrm{a}}$ \\ Ping Li, MD, $\mathrm{PhD}^{\mathrm{c}}$ \\ Xin-liang $\mathrm{Ma}, \mathrm{MD}, \mathrm{PhD}^{\mathrm{d}}$ \\ J. Vinten-Johansen, $\mathrm{PhD}^{\mathrm{a}, \mathrm{b}}$
}

Ischemia-reperfusion damages endothelium and impairs basal production of nitric oxide. Basally released nitric oxide is cardioprotective by its inhibition of neutrophil activities. Loss of endogenous nitric oxide with endothelial injury may occur during two phases: cardioplegic ischemia and reperfusion (aortic declamping). This study tested the hypothesis that inhibition of endogenously released nitric oxide in hearts subjected to regional ischemia, cardioplegic arrest, and reperfusion (1) restricts endogenous cardioprotection and permits neutrophil-mediated damage and (2) expresses damage during the reperfusion phase. L-Nitro-arginine was used to block basal nitric oxide production. In 22 anesthetized dogs, the left anterior descending artery was ligated for 90 minutes followed by 1 hour of arrest with cold multidose (every 20 minutes) blood cardioplegia. Dogs were divided into three groups: the first group received standard unsupplemented blood cardioplegia (group $1, n=8$ ), in the second group L-nitroarginine was administered as an additive to blood cardioplegic solution (1 mmol) and as an infusion during reperfusion ( $34 \mathrm{mg} / \mathrm{kg}$ ) (group $2, n=7$ ), and in the third group L-nitro-arginine was administered only at reperfusion (group 3,n=7). The ligature was released during the second infusion of cardioplegic solution. Infarct size (triphenyltetrazolium chloride) was increased in group 3 (L-nitro-arginine only at reperfusion) compared with that in group 1 (standard blood cardioplegia) $(49 \% \pm 6 \%$ vs $34 \% \pm 2 \%$, respectively), but was not further extended in group 2 ( $\mathrm{L}$-nitro-arginine as an additive to blood cardioplegic solution and at reperfusion) $(56 \% \pm 3 \%$, $p>0.05$ vs group 3 ), which suggests primarily a reperfusion process. Polymorphonuclear neutrophil-specific myeloperoxidase activity in the area at risk was elevated comparably in groups 2 and 3 (group 2: $2.9 \pm 0.5$ units/gm tissue, $p=0.06$ vs group 1; group 3: $3.9 \pm 1.0$ units/gm tissue, $p<$ 0.05 vs group 1) compared with that in the standard blood cardioplegia group (1.7 \pm 0.3 units/gm tissue), suggesting polymorphonuclear neutrophil accumulation occurs primarily during reperfusion. Polymorphonuclear neutrophil adherence in ischemic-reperfused left anterior descending artery segments was comparably greater in group 2 (L-nitro-arginine as an additive to blood cardioplegic solution and at reperfusion: $195 \pm 21$ polymorphonuclear neutrophils $/ \mathrm{mm}^{2}$ of artery, $p<0.05 \mathrm{vs}$ group 1) and group 3 (L-nitro-arginine only at reperfusion: $224 \pm 20$ polymorphonuclear neutrophils $/ \mathrm{mm}^{2}$ of artery, $p<0.05$ vs group 1) relative to that in group 1
From the Departments of Cardiothoracic Surgery a and Physiology/Pharmacology ${ }^{\mathrm{b}}$ and the Hypertension Center, ${ }^{\mathrm{c}}$ Bowman Gray School of Medicine of Wake Forest University, Winston-Salem, N.C., and the Department of Emergency Medicine, ${ }^{\mathrm{d}}$ Jefferson Medical College, Philadelphia, $\mathrm{Pa}$.

Supported in part by grant HLA6179 from the National Heart, Lung, and Blood Institute of the National Institutes of Health.

Read at the Seventy-sixth Annual Meeting of The American Association for Thoracic Surgery, San Diego, Calif., April 28-May 1, 1996.
Received for publication May 6, 1996; revisions requested June 26, 1996; revisions received Sept. 10, 1996; accepted for publication Sept. 12, 1996.

Address for reprints: Jakob Vinten-Johansen, $\mathrm{PhD}$, The Cardiothoracic Research Laboratory, Department of Cardiothoracic Surgery, Carlyle Fraser Heart Center of Emory University, $550 \mathrm{~W}$. Peachtree St. NE, Atlanta, GA 30365-2225.

J Thorac Cardiovasc Surg 1997;113:399-409

Copyright (C) 1997 by Mosby-Year Book, Inc.

$0022-5223 / 97 \$ 5.00+0 \quad \mathbf{1 2 / 6 / 7 8 0 3 3}$ 
$\left(108 \pm 19\right.$ polymorphonuclear neutrophils $/ \mathrm{mm}^{2}$ of artery). There was no significant adherence to nonischemic circumflex arteries. We conclude that blockade of endogenous nitric oxide augments postischemic injury mediated by polymorphonuclear neutrophils, and this damage is expressed primarily during the reperfusion phase. (J Thorac Cardiovasc Surg 1997; 113:399-409)

L ike myocytes, the coronary endothelium falls victim to ischemia-reperfusion injury. Under basal conditions, the intact vascular endothelium actively participates in protection of the vasculature by releasing nitric oxide (NO) synthesized via the $\mathrm{L}^{-}$ arginine-NO synthase pathway, thereby functioning in a homeostatic capacity to prevent thrombus formation and neutrophil adherence. ${ }^{1}$ However, myocardial ischemia and reperfusion impair NO production, ${ }^{2-5}$ thereby compromising this inherent protection. In normal myocardium, blood cardioplegia largely avoids endothelial injury. ${ }^{6,7}$ However, ischemia-reperfusion may injure endothelium. $\mathrm{Na}$ kanishi $^{8}$ and Dignan ${ }^{9}$ and their associates demonstrated that endothelial NO-related injury does not occur after short periods of normothermic ischemia alone, but that significant injury occurs after prolonged ischemia or unmodified blood reperfusion. In ischemic hearts, this endothelial injury is not entirely avoided with unsupplemented blood cardioplegic solutions, ${ }^{8}$ but is reduced by an NO donor (SPM-5185) or L-arginine. ${ }^{10,11}$ However, the importance of basally released endogenous NO in surgical revascularization models has not been investigated.

This study tested the hypotheses that inhibition of endogenously released NO in hearts subjected to regional ischemia, cardioplegic arrest, and reperfusion (1) restricts endogenous cardioprotection by the L-arginine-NO synthase pathway and permits neutrophil-mediated damage and (2) expresses damage during the reperfusion phase in dogs subjected to 1.5 hours of regional ischemia, multidose blood cardioplegia, and reperfusion.

\section{Material and methods}

The dogs were handled in compliance with the "Guide for the Care and Use of Laboratory Animals" published by the National Institutes of Health (NIH Publication No. 85-23, revised 1985). The institutional Animal Care and Use Committee approved the study protocol.

Surgical procedure. Heartworm-free adult mongrel dogs of either sex weighing 18.0 to $34.5 \mathrm{~kg}$ (average 26.3 $\mathrm{kg}$ ) were initially anesthetized with intravenous sodium thiopental $(20 \mathrm{mg} / \mathrm{kg})$ followed by continuous infusion of fentanyl citrate $(0.3 \mu \mathrm{g} / \mathrm{kg}$ per minute) and diazepam $(0.03$. $\mathrm{mg} / \mathrm{kg}$ per minute). Each dog was endotracheally intubated and the lungs ventilated with oxygen-enriched room air to maintain arterial oxygen tension values greater than $100 \mathrm{~mm} \mathrm{Hg}$ with a volume-cycled respirator (Harvard Apparatus, South Natick, Mass.). The right femoral artery and vein were cannulated for arterial blood sampling and fluid administration, respectively. Arterial carbon dioxide tension was maintained between 35 and $45 \mathrm{~mm}$ $\mathrm{Hg}$ by adjustment of ventilatory rate, and $\mathrm{pH}$ was adjusted between 7.37 and 7.43 with administration of intravenous sodium bicarbonate as necessary. After femoral artery cannulation, peripheral blood $(40 \mathrm{ml})$ was collected for subsequent neutrophil isolation (see later section).

Methods used for assessment of regional segmental function by sonomicrometry ${ }^{4}$ and creation of collateraldeficient regional ischemia ${ }^{12}$ have been presented in detail elsewhere. A microdialysis probe ${ }^{13}$ for measurement of in vivo production of $\mathrm{L}$-citrulline, the metabolite of the L-arginine-NO synthase pathway, was inserted into the ischemic-reperfused left anterior descending (LAD) coronary artery. Catheter placement was confirmed at postmortem examination. The dogs were systemically heparinized with $300 \mathrm{U} / \mathrm{kg}$ heparin sodium, supplemented at $300 \mathrm{U} / \mathrm{kg}$ every 90 minutes. The left subclavian artery was cannulated for aortic perfusion. Superior and inferior vena caval cannulas were inserted transatrially into the right atrium and poised in the atrium so as not to impair venous return.

Hemodynamic and segmental function data were obtained at baseline with the circulation intact, after which the LAD and the right ventricular branch of the LAD were transected to divert collateral blood flow from the ischemic zone. After 90 minutes of regional ischemia, hemodynamic and segmental function data were collected again. Cardiopulmonary bypass was instituted as previously described. ${ }^{14}$

Experimental protocol. Dogs were randomly divided into three groups. In the standard blood cardioplegia group (SBCP group, $n=8$ ), unmodified standard blood cardioplegia ${ }^{8}$ was used. In the L-nitro-arginine (L-NA) blood cardioplegia and reperfusion group (LNA-BR group, $n=7$ ), the NO synthase blocker L-NA was added to blood cardioplegic solution (final concentration of $1 \mathrm{mmol} / \mathrm{L}^{11}$ ) and was infused intravenously (34 $\mathrm{mg} / \mathrm{kg}$ body weight) before the aortic crossclamp was released. In the L-NA reperfusion only group (LNA-R group, $n=7$ ), L-NA was infused by intravenous injection $(34 \mathrm{mg} / \mathrm{kg}$ body weight) before the aortic crossclamp was released. A previous study showed that 1 $\mathrm{mmol} / \mathrm{L}$ of $\mathrm{L}-\mathrm{NA}$ blocked the endogenous release of NO stimulated by acetylcholine. ${ }^{11}$ 
Table I. Hemodynamic data at baseline, ischemia, and reperfusion

\begin{tabular}{lccc}
\hline & Baseline & 90 min of ischemia & Reperfusion \\
\hline HR (beats/min) & & & $136 \pm 6$ \\
SBCP & $94 \pm 5$ & $120 \pm 7(0.02)^{*}$ & $136 \pm 12$ \\
LNA-BR & $103 \pm 11$ & $126 \pm 5$ & $135 \pm 9$ \\
LNA-R & $110 \pm 12$ & $136 \pm 10$ & $82 \pm 4$ \\
MAP (mm Hg) & & & $74 \pm 4$ \\
SBCP & $85 \pm 6$ & $84 \pm 4$ & $76 \pm 4$ \\
LNA-BR & $83 \pm 5$ & $81 \pm 7$ & $13.2 \pm 0.9\left(0.03^{*}\right)$ \\
LNA-R & $81 \pm 6$ & $83 \pm 4$ & $11.8 \pm 1.0\left(0.05^{*}\right)$
\end{tabular}

Values represent means plus or minus the standard error of the estimate. $H R$, Heart rate; $M A P$, mean arterial pressure; $P R P$, pressure-rate product. * $p$ Value versus previous period.

Table II. Blood cardioplegic solution delivery volume in milliliters

\begin{tabular}{lccccc}
\hline Group & Warm induction & Cold induction & 20-min infusion & 40 -min infusion & Terminal \\
\hline SBCP & $405 \pm 36$ & $254 \pm 33\left(0.01^{*}\right)$ & $175 \pm 19\left(0.02^{*}\right)$ & $165 \pm 19$ & $275 \pm 51\left(0.01^{*}\right)$ \\
LNA-BR & $372 \pm 31$ & $245 \pm 41\left(0.03^{*}\right)$ & $189 \pm 36\left(0.02^{*}\right)$ & $158 \pm 41$ & $240 \pm 57\left(0.02^{*}\right)$ \\
LNA-R & $369 \pm 23$ & $233 \pm 33\left(0.03^{*}\right)$ & $215 \pm 15$ & $165 \pm 22$ & $281 \pm 31\left(0.01^{*}\right)$ \\
\hline
\end{tabular}

Values are given as means plus or minus the standard error of the estimate.

${ }^{*} p$ Value versus previous period.

Cardioplegic arrest. After 90 minutes of LAD occlusion, the aorta was crossclamped, and blood cardioplegic solution was delivered intermittently (every 20 minutes) at $50 \mathrm{~mm} \mathrm{Hg}$ pressure by the warm-cold induction modality ${ }^{15}$ with terminal warm infusion. Before the 20-minute delivery of cardioplegic solution, the LAD vessel loop was removed and the arteriotomy for collateral diversion was closed. Systemic temperature was maintained at $28^{\circ} \mathrm{C}$.

At the end of 1 hour of arrest, systemic rewarming to $37^{\circ} \mathrm{C}$ was achieved, and the crossclamp was removed. Mean aortic pressure was gradually increased from $50 \mathrm{~mm}$ $\mathrm{Hg}$ to $80 \mathrm{~mm} \mathrm{Hg}$ after electromechanical reanimation. The heart was maintained in vented bypass for the initial 30 minutes of reperfusion. After systemic blood $\mathrm{Ca}^{2+}$ content was corrected to baseline values, ${ }^{16}$ the dog was weaned off bypass and allowed to support the circulation for an additional 30 minutes in the working state. After postischemic measurements were made, the heart was excised and immediately immersed in cold Krebs-Henseleit solution with the following composition (in millimolars per liter): $118 \mathrm{NaCl}, 4.7 \mathrm{KCl}, 1.2 \mathrm{KH}_{2} \mathrm{PO}_{4}, 1.2$ $\mathrm{MgSO}_{4}, 2.5 \mathrm{CaCl}_{2}, 12.5 \mathrm{NaHCO}_{3}$, and 10 glucose. The ischemic-reperfused LAD and the nonischemic left circumflex coronary artery (LCx) were carefully isolated, and artery segments were removed and placed in cold, oxygenated Krebs-Henseleit solution.

Determination of area at risk and infarct size. The interrupted $\mathrm{LCx}$ was bridged with a polyethylene tube to reestablish continuity, and gentian violet dye was perfused through the aortic root to stain the normally perfused region blue. The area at risk was demarcated by the lack of blue staining. The area of necrosis and infarct size were determined by triphenyltetrazolium chloride vital stain and gravimetric analysis as previously described. ${ }^{4,17}$

Data acquisition and analysis. Hemodynamic data were acquired and processed by computer as detailed previously. ${ }^{4,18}$ Hemodynamic and cardiodynamic data were averaged from no fewer than 15 beats. The pressurerate product, percent segmental shortening, segmental work, and diastolic segmental stiffness were determined as previously described. ${ }^{4}$

In vitro coronary artery studies. Plasma creatine kinase activity, cardiac myeloperoxidase activity, and coronary reactivity were measured as described previously. ${ }^{11,12}$ In addition, the heart was excised from five normal dogs to obtain control data. Neutrophils were isolated and adherence to the coronary artery segments was determined as previously detailed. ${ }^{\text {is }}$

In vivo estimation of local NO production. Estimation of NO production by cardiac microdialysis was modified from the method described by Northington and associates ${ }^{20}$ in which L-arginine is converted to an equimolar concentration of L-citrulline and NO by the action of NO synthase. Three micromolars of L- $\left[{ }^{14} \mathrm{C}\right]$ arginine (Dupont/ NEN, Boston) was prepared in fresh Krebs-Henseleit buffer. After 1 hour of stabilization, the microdialysis buffer was replaced with $\mathrm{L}-\left[{ }^{14} \mathrm{C}\right]$ arginine solution and constantly perfused at $2 \mu \mathrm{l} / \mathrm{min}$. Dialysate samples were collected every 30 minutes ( $60 \mu \mathrm{l}$ in each sample) and diluted in $200 \mu \mathrm{l}$ of $30 \mathrm{mmol} N$-2-hydroxyethylpiperazine$N^{\prime}$-2-ethane-sulfonic acid buffer (pH 5.5) containing 3 mmol ethylenediaminetetraacetic acid and applied to a chromatography column of $0.5 \mathrm{ml}$ Dowex AG-50WX8 resin $\left(\mathrm{Na}^{+}\right.$form, $\mathrm{pH}$ 7.0, Sigma Chemical Co., St. Louis, 

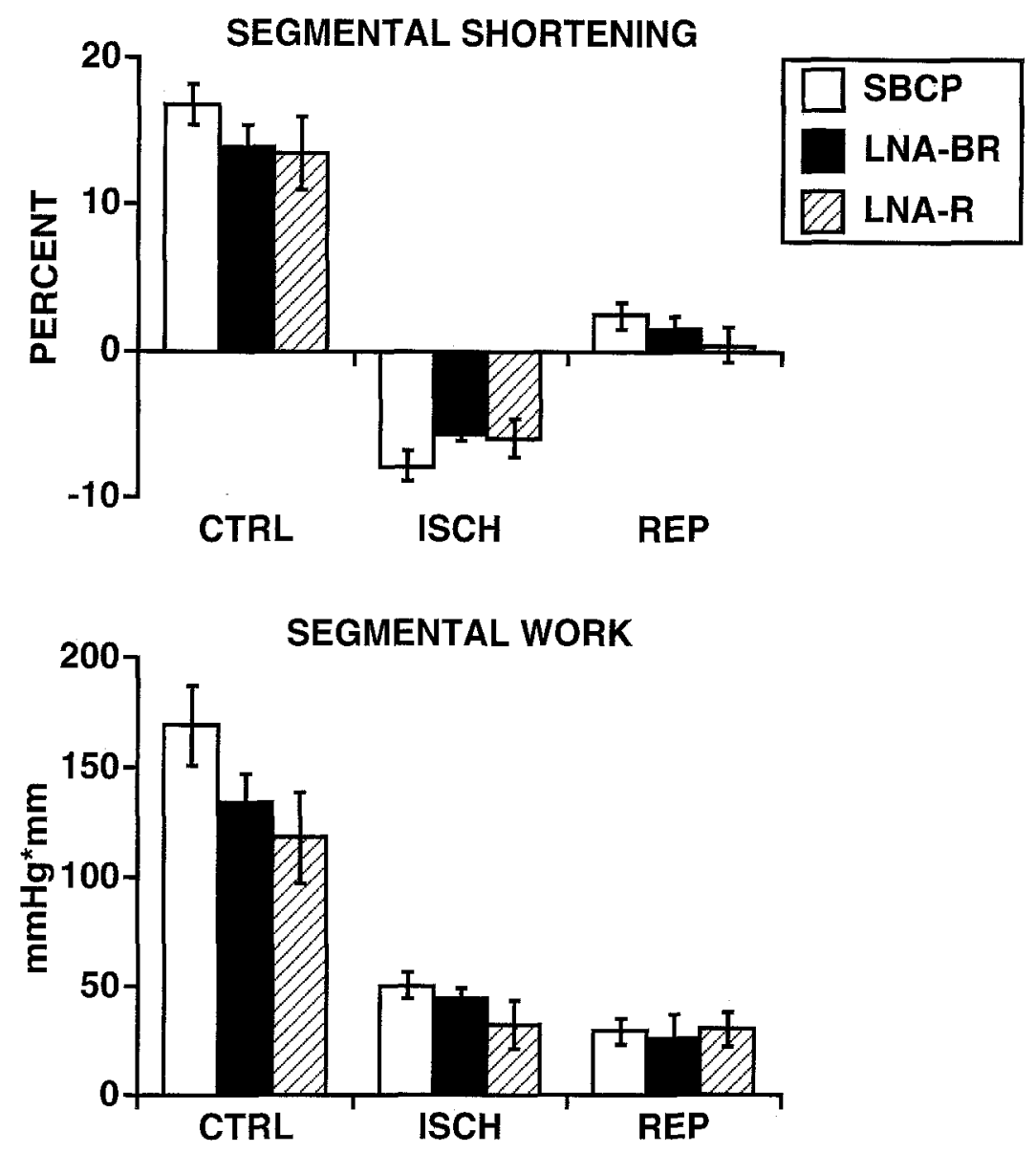

Fig. 1. Segmental shortening (top panel) and segmental work (bottom panel) in ischemic-reperfused zone at baseline (CTRL, control), at the end of coronary occlusion (ISCH, ischemia), and after 60 minutes of reperfusion (REP) in the working heart. Bar heights represent mean plus or minus the standard error of the mean. There were no significant differences among the three groups.

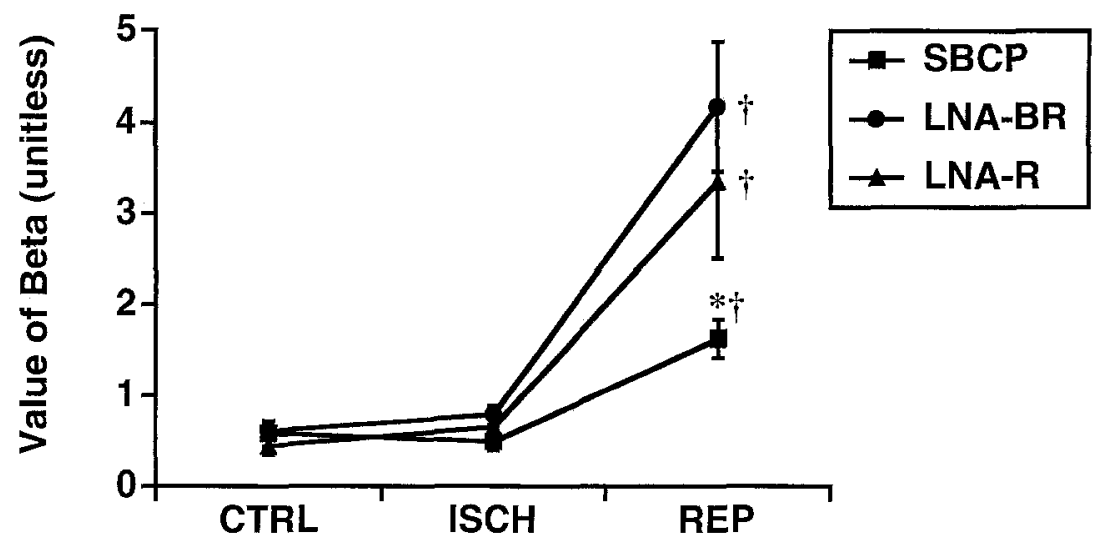

Fig. 2. Segmental stiffness, indexed as $\beta$-coefficient or modulus of stiffness derived from the exponential end-diastolic pressure-segment length relationship. Value of $\beta$ in SBCP group was significantly less than that in the other two groups at reperfusion. Symbol heights represent mean plus or minus the standard error of the mean. CTRL, Control; $I S C H$, ischemia; $R E P$, reperfusion. ${ }^{*} p<0.05$ versus other two groups; $\dagger p<$ 0.05 versus previous value. 
Table III. Segmental length data for ischemic-reperfused segment

\begin{tabular}{cccc}
\hline & Baseline & 90 min of ischemia & Reperfusion \\
\hline EDL (mm) & & & $13.1 \pm 1.1\left(0.03^{*}\right)$ \\
SBCP & $13.0 \pm 1.1$ & $16.5 \pm 1.4\left(0.03^{*}\right)$ & $14.5 \pm 1.5\left(0.04^{*}\right)$ \\
LNA-BR & $15.1 \pm 1.5$ & $18.5 \pm 1.4\left(0.03^{*}\right)$ & $12.1 \pm 0.8\left(0.04^{*}\right)$ \\
LNA-R & $12.5 \pm 0.7$ & $15.3 \pm 0.7\left(0.04^{*}\right)$ & \\
ESL (mm) & & & $12.8 \pm 1.1\left(0.03^{*}\right)$ \\
SBCP & $10.9 \pm 1.0$ & $17.8 \pm 1.4\left(0.01^{*}\right)$ & $14.3 \pm 1.5\left(0.02^{*}\right)$ \\
LNA-BR & $13.0 \pm 1.3$ & $19.5 \pm 1.4\left(0.02^{*}\right)$ & $12.1 \pm 0.8\left(0.03^{*}\right)$
\end{tabular}

Values are given as means plus or minus the standard error of the estimate. $E D L$, End-diastolic length; $E S L$, end-systolic length.

" $p$ Value versus previous period.

Table IV. Plasma creatine kinase activity (international units per gram protein) during the time course of the experiment

\begin{tabular}{lccccc} 
& & & \multicolumn{2}{c}{ Reperfusion } \\
Group & Preischemia & Ischemia & Blood cardioplegia & $B E$ & $B W$ \\
\hline SBCP & $1.15 \pm 0.29$ & $3.77 \pm 0.64^{*}$ & $6.35 \pm 0.89^{*}$ & $10.93 \pm 1.35^{*}$ & $17.33 \pm 1.96^{*} \dagger$ \\
LNA-BR & $1.32 \pm 0.40$ & $7.30 \pm 1.65^{* \dagger}$ & $13.63 \pm 3.92^{*}+$ & $24.32 \pm 7.24$ & $38.63 \pm 9.78^{*}$ \\
LNA-R & $1.09 \pm 0.21$ & $4.04 \pm 0.43^{*}$ & $7.40 \pm 1.01^{*}$ & $18.81 \pm 3.69^{*}$ & $29.96 \pm 3.44^{*}$ \\
\hline
\end{tabular}

Values are given as means plus or minus the standard error of the estimate. $B E$, Beating empty; $B W$, beating working.

${ }^{*} p<0.05$ versus previous period.

$\dot{\phi} p<0.05$ versus other two groups.

$\grave{i} p<0.05$ versus SBCP group.

Mo.). $L-\left[{ }^{14} \mathrm{C}\right]$ citrulline was eluted with $2 \mathrm{ml}$ buffer and quantified by liquid scintillation (LKB 1219, Rakebete, Md.). $\mathrm{L}-\left[{ }^{14} \mathrm{C}\right]$ citrulline activity obtained from each dialysate sample was expressed as percent of baseline value.

Statistical analysis. All data were analyzed with the Statistical Analysis System program (PC-SAS, SAS Institute, Cary, N.C.). Time-related differences and grouptime interactions were analyzed by two-way analysis of variance for repeated measures. Infarct size and myeloperoxidase activity were analyzed by one-way analysis of variance. Vascular responses were expressed as percent changes of tension from the precontracted levels and compared by one-way analysis of variance. The dose of the drug required to effect relaxation to $50 \%$ of maximum precontracted levels $\left(\mathrm{EC}_{50}\right)$ was calculated and expressed as the negative log of the drug concentration. All data are presented as means plus or minus the standard error of the mean.

\section{Results}

Twenty-two dogs (SBCP group, 8; LNA-BR group, 7; LNA-R group, 7) were entered into the final data analysis of infarct size and regional function.

Hemodynamics, myocardial temperature, and cardioplegic solution volume. Blood gas data before bypass and after stabilization on bypass were not significantly different among the groups. There were no group differences in the number of cardioversions required during the early period of reper- fusion (SBCP group, 5; LNA-BR group, 4; LNA-R group, 5).

Hemodynamic data for the three groups at baseline and during ischemia and reperfusion are summarized in Table I. There were no group differences in any of the parameters at baseline. During coronary occlusion, heart rate was significantly increased from the baseline value only in the SBCP group. However, there were no group differences in any of the parameters during ischemia. During reperfusion, pressure rate product increased significantly from values during ischemia in the SBCP and LNA-BR groups, but with no group differences at that time.

There were no significant differences among the three groups in blood cardioplegic solution composition ( $\mathrm{pH}$, oxygen tension, osmolality, hematocrit), delivery volume, or myocardial temperatures (Table II).

Segmental systolic and diastolic function. There were no group differences in segmental systolic or diastolic function at baseline (Table III). Ischemia caused paradoxic systolic expansion so that the end-systolic length exceeded the end-diastolic length in all groups. Both the magnitude of segmental shortening and segmental work (Fig. 1) showed similar dyskinesis in all three groups. Segmental 

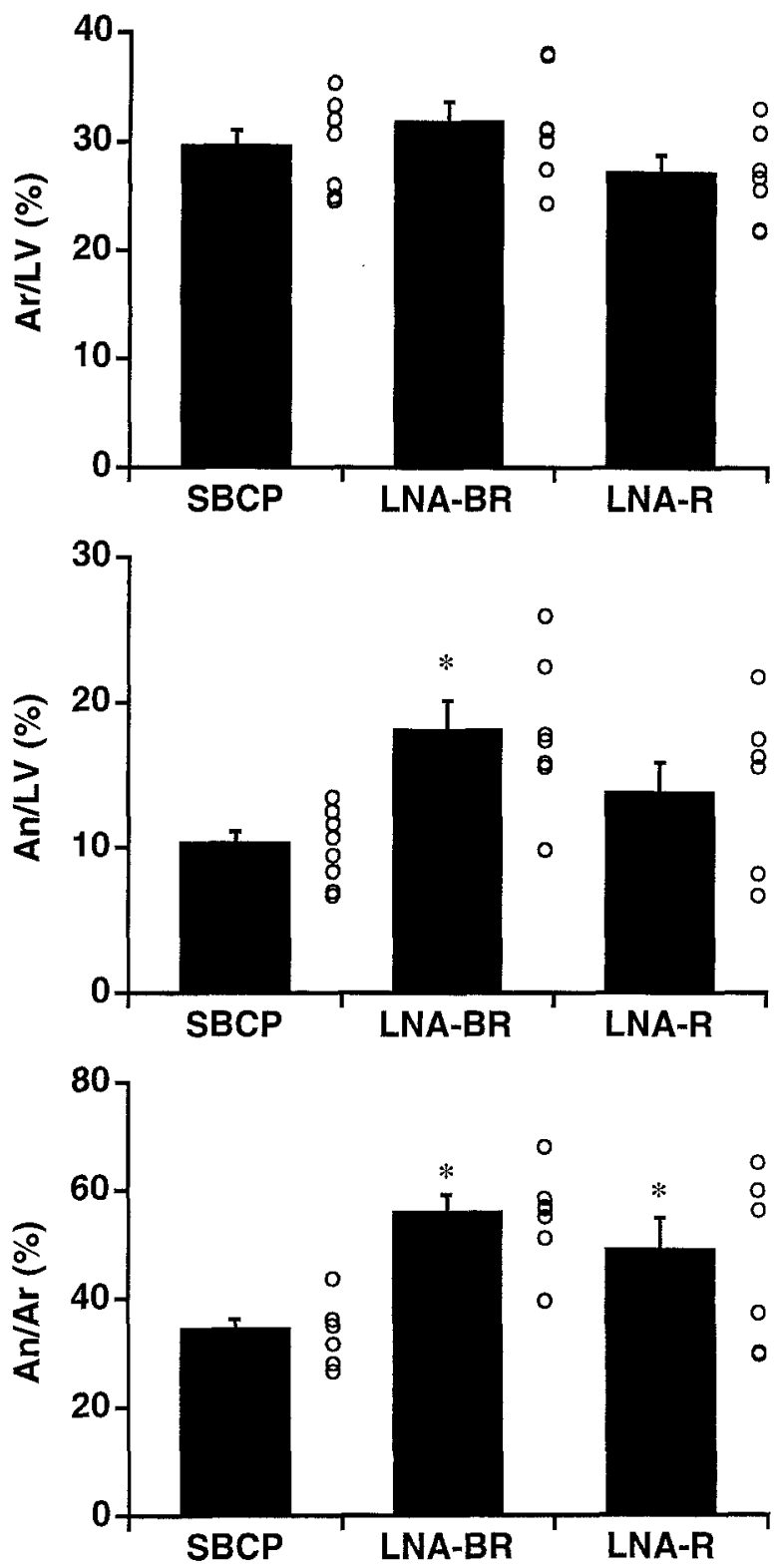

Fig. 3. Mass of the area at risk versus mass of left ventricle as percentage ( $A r / L V$, top panel), mass of the area of necrosis versus mass of left ventricle as percentage (An/LV, middle panel), and area of necrosis as percent of area at risk (An/Ar, bottom panel). Although the area placed at risk was comparable among groups, the areas of necrosis as percent of area at risk was significantly increased in LNA-BR and LNA-R groups relative to that in SBCP group. Bars represent mean plus or minus the standard error of the mean; circles represent individual values for each group. ${ }^{*} p<0.05$ versus SBCP group. shortening and work were depressed during reperfusion, with no group differences.

The $\beta$-coefficient (modulus of diastolic segmental stiffness) data of the exponential end-diastolic pressure-segment length relation are summarized in Fig. 2. LAD occlusion did not increase the segmental stiffness in the area at risk to values greater than baseline in any of the three groups. During reperfusion, segmental stiffness was significantly increased to greater than that during ischemia in all three groups. However, the $\beta$-coefficients in the LNA-BR and LNA-R groups were significantly greater than those in the SBCP group.

Area at risk and infarct size. The masses of the left ventricle and area at risk were similar among the groups, as was the percentage area at risk (Fig. 3, top panel). The area of necrosis, expressed as a percentage of the area at risk, shown in Fig. 3, bottom panel, was significantly increased in both L-NA-treated groups compared with the SBCP group. Therefore blockade of the endogenous L-arginine-NO pathway by L-NA exacerbated infarct size presumably by inhibiting production of endogenous NO.

Plasma creatine kinase activity. There was no significant difference in plasma creatine kinase activity at baseline among the three groups (Table IV). All three groups showed a significant increase in plasma creatine kinase activity during LAD occlusion to the end of the experiment. Creatine kinase activity increased dramatically during the cardioplegia period in the three groups, but was significantly greater in the LNA-BR and LNA-R groups than in the SBCP group. The significantly greater creatine kinase activities in the two groups that received $\mathrm{L}$ NA were consistent with the larger infarct sizes relative to those in the SBCP group.

Cardiac myeloperoxidase activity. Myeloperoxidase activity in the nonischemic zone (Fig. 4) was comparably low among the SBCP, LNA-BR, and LNA-R groups. Myeloperoxidase activity in the ischemic but nonnecrotic zone increased significantly over values in the nonischemic zone in the three groups. However, myeloperoxidase activity in the LNA-R group was significantly greater by $132.4 \%$ than that in the SBCP group. Also, there was a strong tendency $(p=0.06)$ for the LNA-BR group to have greater myeloperoxidase activity compared with that in the SBCP group. However, in the necrotic zone, myeloperoxidase activity in the three groups was less than that in the ischemic nonnecrotic zone without differences among the three groups. 


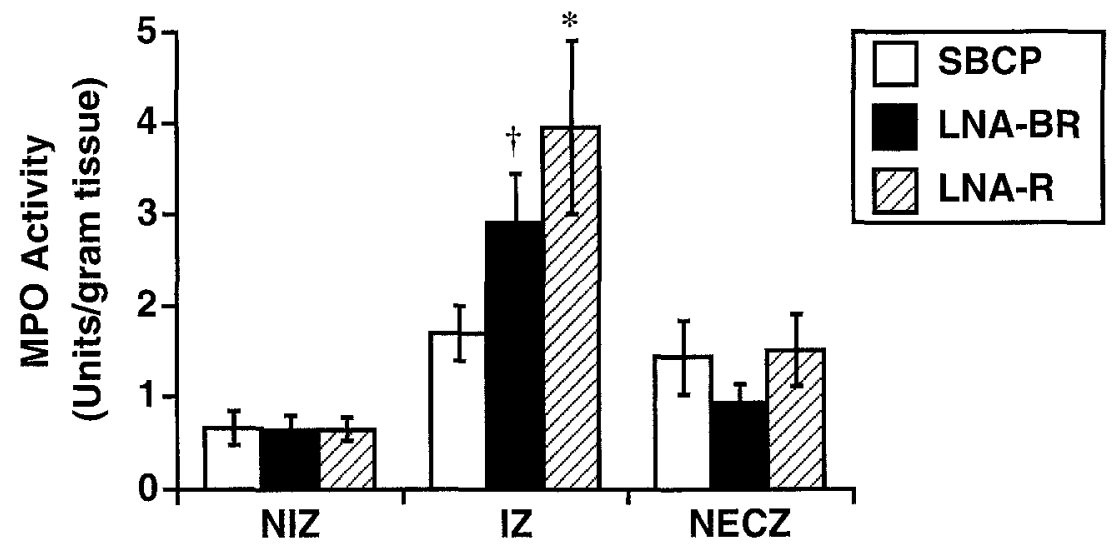

Fig. 4. Myeloperoxidase (MPO) activity in nonischemic zone (NIZ), ischemic but nonnecrotic zone (IZ), and ischemic-necrotic zone (NEC). Myeloperoxidase activity was significantly less in SBCP group in ischemic but nonnecrotic zone. Bar heights represent mean plus or minus the standard error of the mean. ${ }^{*} p<0.05$ versus $\mathrm{SBCP}$ group; $\uparrow p=0.06$ versus SBCP group.

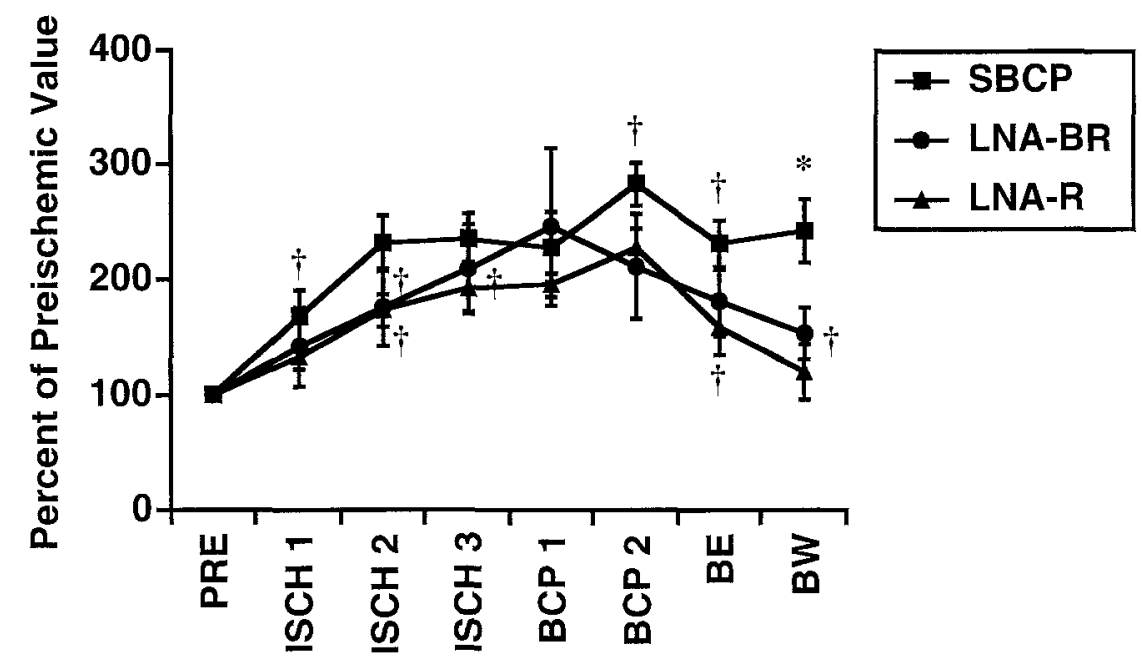

Fig. 5. Dialysate interstitial $\mathrm{L}-\left[{ }^{14} \mathrm{C}\right]$ citrulline concentration in area at risk (percent change of control value). Citrulline level was significantly higher in SBCP group at the end of reperfusion. Symbol heights represent mean plus or minus the standard error of the mean. PRE, Preischemia; ISCH1, first 30 minutes of occlusion; ISCH2, 30 to 60 minutes of occlusion; ISCH3, 60 to 90 minutes of occlusion; $B C P 1$, first 30 minutes of blood cardioplegic arrest; $B C P 2,30$ to 60 minutes of blood cardioplegic arrest; $B E, 30$ minutes of beating empty period; $B W, 30$ minutes of beating working period. * $p<0.05$ versus other two groups; $\dagger p$ $<0.05$ versus previous period.

In vivo local NO production. $\mathrm{L}-\left[{ }^{14} \mathrm{C}\right]$ citrulline activity obtained from intramyocardial microdialysis was expressed as a percent of the preischemic value and results are shown in Fig. 5. During the coronary occlusion, local NO production was significantly increased in all three groups with no group differences. During blood cardioplegic arrest, the L$\left[{ }^{14} \mathrm{C}\right]$ citrulline levels tended to decrease in the two groups receiving L -NA. During reperfusion, the L$\left[{ }^{14} \mathrm{C}\right]$ citrulline level in the L-NA-treated groups was significantly less than that in the SBCP group. These data confirm that L-NA inhibited NO synthase activity in the treated groups.

In vitro coronary artery data

Neutrophil adherence assay. Adherence of unstimulated polymorphonuclear leukocytes to isch- 


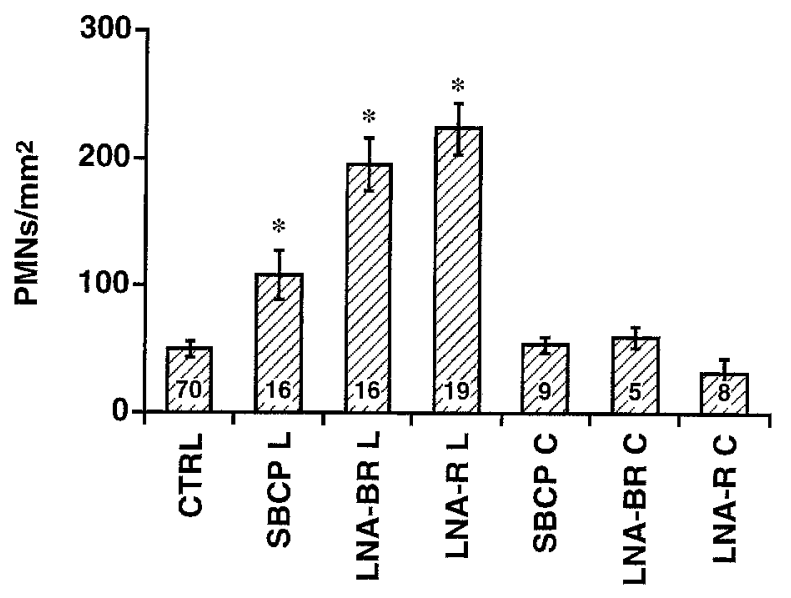

Fig. 6. Adherence of unactivated polymorphonuclear leukocytes $(P M N S)$ to the endothelium of normal coronary arterial ring segments (CTRL), ischemic-reperfused LAD ring segments from each group (SBCP $L, L N A-B R$ $L, L N A-R L$, where $L$ indicates $L A D$ ), and LCX ring segments from each group ( $S B C P C, L N A-B R C, L N A-R$ $C$, where $C$ indicates $\mathrm{LCx}$ ). Polymorphonuclear leukocyte adherence to LAD segments was significantly increased in all three groups. However, adherence in L-NA-treated groups was significantly higher than that in the SBCP group. There was no increase in adherence and no group differences in LCx ring segments. Bar heights represent mean plus or minus the standard error of the mean; numbers in bars represent numbers of coronary arterial ring segments studied. ${ }^{*} p<0.05$ versus other groups.

emic-reperfused coronary artery segments is used as an index of basal NO release. When unstimulated polymorphonuclear leukocytes were coincubated with normal unstimulated coronary endothelium as a control observation, very little adherence of neutrophils was observed (Fig. 6). However, in the ischemic-reperfused LAD segments, neutrophil adherence was significantly increased in the SBCP group, suggesting loss of NO production by the endothelium. In addition, L-NA treatment significantly increased neutrophil adherence further compared with that in the control LAD segment and the SBCP group. In contrast, adherence to nonischemicreperfused LCx segments from each group was similar to that in unstimulated control arteries (Fig. 6).

Agonist-stimulated coronary artery relaxation data. The optimal passive length determined from lengthtension responses to potassium chloride in the LAD and $\mathrm{LCx}$ was comparable among all groups.

Endothelium-dependent relaxations. Fig. 7 shows vasodilator responses to acetylcholine (upper panel) in the LAD and LCx rings. The concentration response curve in LAD rings in the SBCP group was shifted markedly to the right compared with that in the control group, involving a decrease in maximal response and an increase in the $\mathrm{EC}_{50}(6.99 \pm 0.05 \mathrm{vs}$ $7.68 \pm 0.03-\log [\mathrm{mol}]$ in control group). However, the shift in the curves for the LNA-BR $(6.26 \pm 0.04)$ and LNA-R (6.44 \pm 0.05$)$ groups was significantly greater than that in the SBCP group. The concentration response curves to acetylcholine in the $\mathrm{LCx}$ rings from all three groups were also markedly shifted to the right relative to those in the control group. The rightward shift in the SBCP group was more modest $(7.29 \pm 0.15)$ than that in rings from the LAD in the same group. However, marked shifts indicative of endothelial damage occurred in the LNA-BR $(6.23 \pm 0.06)$ and LNA-R $(6.27 \pm 0.06)$ groups relative to control LCx values $(7.68 \pm 0.03)$.

The concentration responses to A23187 in LAD and $\mathrm{LCx}$ rings were similar in both $\mathrm{L}-\mathrm{NA}$-treated groups to those observed for acetylcholine (Fig. 7, center panel) except that the rightward shift for the SBCP group was less pronounced. There were no group differences in the maximum relaxation among all four groups. $\mathrm{EC}_{50}$ values in the $\mathrm{LAD}$ rings were significantly greater in the SBCP $(7.65 \pm 0.07$ $-\log [\mathrm{mol}])$, LNA-BR $(7.05 \pm 0.09)$, and LNA-R $(7.14 \pm 0.04)$ groups than in the control arteries $(7.84 \pm 0.04)$, consistent with a rightward shift. $\mathrm{EC}_{50}$ values in the LCx rings were similar in the control $(7.84 \pm 0.04)$ and SBCP $(7.79 \pm 0.07)$ groups and increased in the LNA-BR (7.26 \pm 0.04$)$ and LNA-R $(7.33 \pm 0.06)$ groups, consistent with the greater rightward shift in these two groups.

Endothelium-independent smooth-muscle relaxations. Acidified sodium nitrite was used to induce endothelium-independent relaxation (Fig. 7, bottom panel). The concentration response curves in LAD rings from the $\mathrm{SBCP}\left(\mathrm{EC}_{50}\right.$ values $5.04 \pm 0.06$ $-\log [\mathrm{mol}])$, LNA-BR $(4.77 \pm 0.06)$, and LNA-R $(4.99 \pm 0.07)$ groups were markedly shifted to the right compared with the curve from the control group $(5.49 \pm 0.05)$, without a reduction in the maximum relaxation compared with that in the control group. However, the shift was significantly greater in the LNA-BR group than in the SBCP and LNA-R groups. In the LCx rings, there were no significant differences in the relaxation responses among all three groups $\left(\mathrm{EC}_{50}\right.$ in SBCP group, $5.41 \pm 0.12$; LNA-BR group, $5.20 \pm 0.09$; LNA-R group, $5.37 \pm 0.11$ ) compared with control values $(5.49 \pm 0.05)$. 

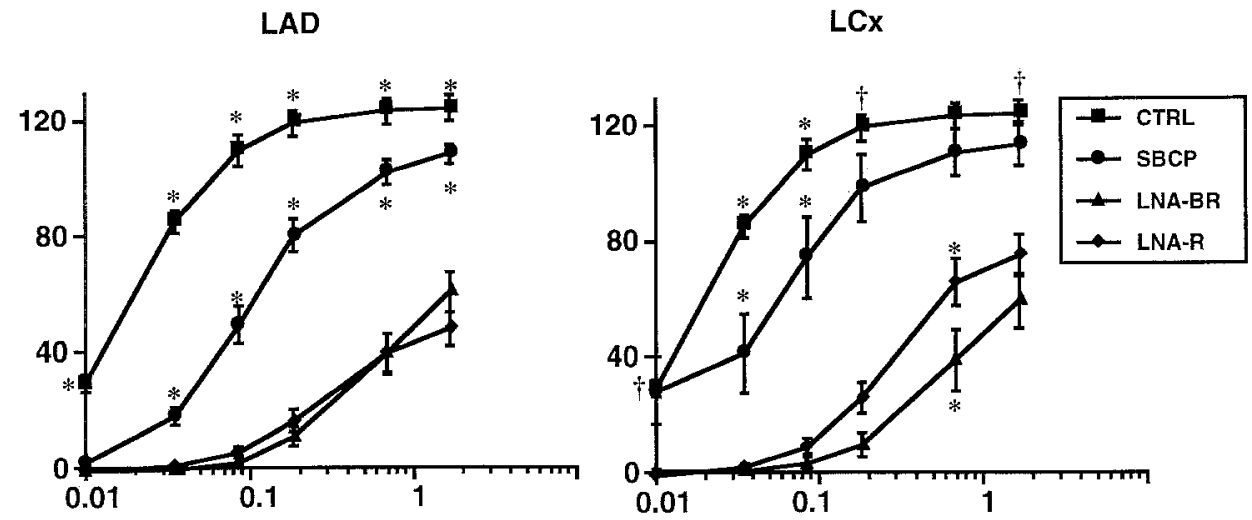

Acetylcholine $[\mu \mathrm{M}]$
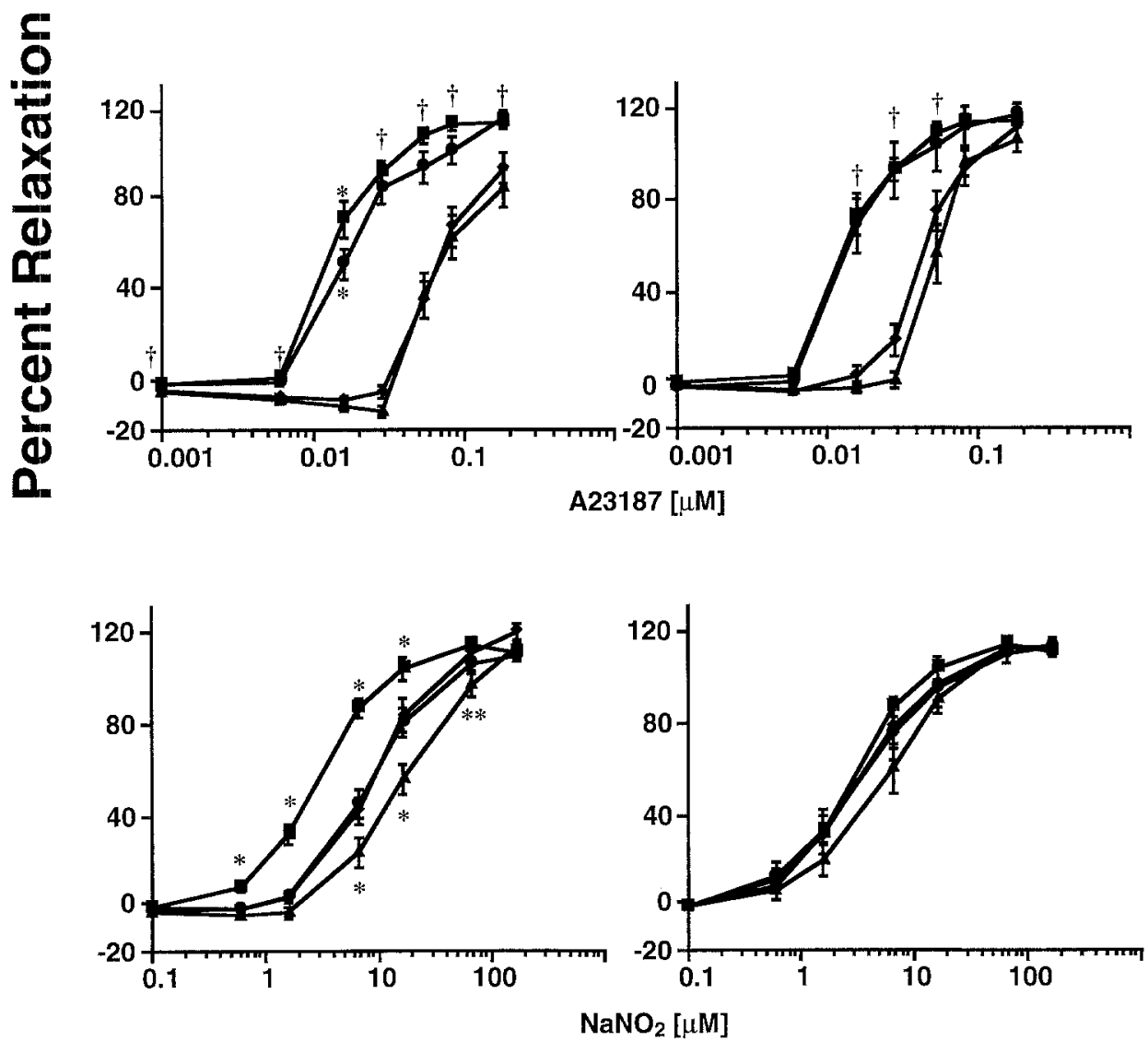

Fig. 7. Vasodilator responses in LAD and LCx vascular rings to acetylcholine, the receptor-dependent, endothelium-dependent stimulator of NO (upper panel); receptor-independent, endothelium-dependent NO-stimulator calcium ionophore A23187 (middle panel); and smooth-muscle relaxing NO donor agent acidified $\mathrm{NaNO}_{2}$ (bottom panel). Relaxation responses were expressed as percentage of U46619-induced precontraction. Symbol heights represent mean plus or minus the standard error of the estimate. ${ }^{*} p<0.05$ versus other three groups; ${ }^{* *} p<0.05$ LNA-BR group versus control (CTRL) group; $\dagger p<0.05$ control and SBCP groups versus LNA-BR and LNA-R groups. 


\section{Discussion}

In the present study, we found that inhibition of endogenous NO release increased infarct size by $62.5 \%$ in the LNA-BR group (combination of blood cardioplegia and reperfusion) and by $42.6 \%$ in the LNA-R group (reperfusion only), compared with results with an unsupplemented blood cardioplegic solution. In addition, L-NA treatment increased diastolic segmental stiffness in the ischemic-reperfused segment, increased neutrophil accumulation in the ischemic-reperfused myocardium, and exacerbated neutrophil adherence to the ischemic-reperfused coronary artery segment; consistent with a loss of basal NO release. L-NA reduced basal endothelial function in ischemic-reperfused LAD segments but not in normal LCx segments. Our data suggest that the actions of L-NA were exerted primarily during blood reperfusion (aortic declamping) because the LNA-BR group did not significantly extend ischemia-reperfusion injury further compared with that in the LNA-R group.

Both basal endothelial function and stimulated vasodilator responses were depressed in ischemicreperfused LAD segments. The depression in agonist-stimulated endothelial vasodilator responses was modest in the standard unsupplemented blood cardioplegia group and may reflect some degree of smooth muscle damage (Fig. 7). Endothelium-dependent vasodilator responses were more severely depressed in the L-NA-treated groups. Although this observation is consistent with ischemia-reperfusion-induced endothelial damage reported in other studies, $5,8,10,11$ similar results may be observed with a persistent effect of L-NA independent of endothelial injury. We have previously observed a blunted agonist (acetylcholine)-stimulated relaxation response in control coronary arteries treated with L-NA, despite a prolonged washout period of up to 2 to 3 hours. ${ }^{11}$ Blunted responses were also observed in the LCx in the present study, although these vessels encountered only global cardiac arrest. It is possible that L-NA could cause a persistent or irreversible inhibition of agonist-stimulated NO release while having no effect on basal NO. The exact mechanism of this effect is not known but may involve (1) different compartments of basal and pharmacologically stimulated NO, (2) a limitation of stimulated NO release to that required to achieve basal levels, or (3) a partial inhibition of the muscarinic receptor by L-NA. Therefore, in this study, the agonist stimulator response assay cannot discriminate between NO synthase inhibition with L-NA or endothelial injury in the circumflex coronary arteries.

In summary, the present study demonistrates that inhibition of endogenous NO synthase activity by L$\mathrm{NA}$ administered in blood cardioplegic solution and during reperfusion, or during reperfusion only, is associated with a significant increase in infarct size and regional diastolic dysfunction. Also, L-NA treatment was associated with an increase in neutrophil accumulation within the area at risk. Inhibition of NO synthase-mediated protection may have also damaged both the coronary vascular endothelium and to a lesser extent the vascular smooth muscle. These data are consistent with the hypothesis that endogenous NO participates in the inherent protection of the ischemic-reperfused and the normal myocardium during cardiac operations. These data provide a rationale for implementing proactive protective strategies that target protection of the vascular endothelium.

\section{REFERENCES}

1. Ma X, Weyrich AS, Lefer DJ, Lefer AM. Diminished basal nitric oxide release after myocardial ischemia and reperfusion promotes neutrophil adherence to coronary endothelium. Circ Res 1993;72:403-12.

2. VanBenthuysen KM, McMurtry IF, Horwitz LD. Reperfusion after acute coronary occlusion in dogs impairs endothelium-dependent relaxation to acetylcholine and augments contractile reactivity in vitro. J Clin Invest 1987;79:265-74.

3. Tsao PS, Aoki N, Lefer DJ, Johnson G III, Lefer AM. Time course of endothelial dysfunction and myocardial injury during myocardial ischemia and reperfusion in the cat. Circulation 1990;82:1402-12.

4. Nakanishi K, Vinten-Johansen J, Lefer DJ, et al. Intracoronary $L$-arginine during reperfusion improves endothelial function and reduces infarct size. Am J Physiol 1992;263: H1650-8.

5. Pearson PJ, Lin PJ, Schaff HV. Global myocardial ischemia and reperfusion impair endothelium-dependent relaxations to aggregating platelets in the canine coronary artery: a possible cause of vasospasm after cardiopulmonary bypass. $\mathrm{J}$ Thorac Cardiovasc Surg 1992;103:1147-54.

6. Sellke FW, Shafique T, Johnson RG, et al. Blood and albumin cardioplegia preserve endothelium-dependent microvascular responses [see comments]. Ann Thorac Surg 1993;55:977-85.

7. Nilsson FN, Miller VM, Vanhoutte PM, McGregor CGA. Methods of cardiac preservation alter the function of the endothelium in porcine coronary arteries. J Thorac Cardiovasc Surg 1991;102:923-30.

8. Nakanishi K, Zhao Z-Q, Vinten-Johansen J, Lewis JC, McGee DS, Hammon JW Jr. Coronary artery endothelial dysfunction after ischemia, blood cardioplegia, and reperfusion. Ann Thorac Surg 1994;58:191-9.

9. Dignan RJ, Dyke CM, Abd-Elfattah AS, et al. Coronary artery endothelial cell and smooth muscle dysfunction after global myocardial ischemia. Ann Thorac Surg 1992;53:311-7. 
10. Nakanishi K, Zhao Z-Q, Vinten-Johansen J, Hudspeth DA, McGee DS, Hammon JW Jr. Blood cardioplegia enhanced with the nitric oxide donor SPM-5185 counteracts postischemic endothelial and ventricular dysfunction. J Thorac Cardiovasc Surg 1995;109:1146-54.

11. Sato H, Zhao Z-Q, McGee DS, Williams MW, Hammon JW Jr, Vinten-Johansen J. Supplemental L-arginine during cardioplegic arrest and reperfusion avoids regional postischemic injury. J Thorac Cardiovasc Surg 1995;110:302-14.

12. Lefer DJ, Nakanishi K, Johnston WE, Vinten-Johansen J. Antineutrophil and myocardial protection actions of a novel nitric oxide donor after acute myocardial ischemia and reperfusion in dogs. Circulation 1993;88:2337-50.

13. Van Wylen DGL, Willis J, Sodhi J, Weiss RJ, Lasley RD, Mentzer RM Jr. Cardiac microdialysis to estimate interstitial adenosine and coronary blood flow. Am J Physiol 1990;258: H1642-9.

14. Hudspeth DA, Williams MW, Zhao Z-Q, et al. Pentostatinaugmented interstitial adenosine prevents postcardioplegia injury in damaged hearts. Ann Thorac Surg 1994;58:719-27.

15. Rosenkranz ER, Vinten-Johansen J, Buckberg GD, Okamoto $\mathrm{F}$, Edwards H, Bugyi H. Benefits of normothermic induction of blood cardioplegia in energy-depleted hearts, with maintenance of arrest by multidose cold blood cardioplegic infusions. J Thorac Cardiovasc Surg 1982;84:66777.

16. Yokoyama H, Julian JS, Vinten-Johansen J, et al. Postischemic $\left[\mathrm{Ca}^{2+}\right]$ repletion improves cardiac performance without altering oxygen demands. Ann Thorac Surg 1990;49:894-902.

17. Vinten-Johansen $\mathbf{J}$, Lefer DJ, Nakanishi K, Johnston WE, Brian CA, Cordell AR. Controlled coronary hydrodynamics at the time of reperfusion reduces postischemic injury. Coron Artery Dis 1992;3:1081-93.

18. Vinten-Johansen J, Nakanishi K, Zhao Z-Q, McGee DS, Tan P. Acadesine improves surgical myocardial protection with blood cardioplegia in ischemically injured canine hearts. Circulation 1993;88:II350-8.

19. Sato H, Zhao Z-Q, Vinten-Johansen J. L-Arginine inhibits neutrophil adherence and coronary artery dysfunction. Cardiovasc Res 1996;31:63-72.

20. Northington FJ, Tobin JR, Koehler RC, Traystman RJ. In vivo production of nitric oxide correlates with NMDAinduced cerebral hyperemia in newborn sheep. Am J Physiol 1995;269:H215-21.

\section{Discussion}

Dr. Robert A. Guyton (Atlanta, $G a$.). I would like a little clarification of the time course of the neutrophil adher- ence. The proponents of leukocyte depletion claim that a temporary depletion of leukocytes will prevent damage to the ischemic area because the vessels are restored to normal and leukocyte adherence is not increased after 10 or 15 minutes. What was the time course of the testing of these isolated arteries?

Dr. Sato. The coronary arteries were excised from the area at risk representing ischemic-reperfused vessels and from the nonischemic zone at the end of reperfusion in each experiment. The postexperimental coronary artery segments were then incubated with autologous neutrophils obtained from the same animal the day of the experiment. Postreperfusion neutrophil adherence to the endothelium of these coronary arteries is therefore an index of damage to basal NO release by the endothelium.

Dr. Guyton. These arteries were excised and tested after 60 minutes of reperfusion?

Dr. Sato. Yes, this was done after reperfusion, at the end of the experiment.

Dr. Neil K. Worrall (St. Louis, Mo.). Agents such as nitro-arginine are very potent constitutive NO synthase inhibitors and therefore act as vasoconstrictors, and in this study, at the doses that were used, there probably would have been a reduction in blood flow to the myocardium. Did the authors measure blood flow in the study?

Dr. Sato. No, we did not measure myocardial blood flow. We did measure blood cardioplegic solution volumes to determine whether vasoconstriction induced by $N^{\omega}$ nitro-L-arginine methyl ester may have impaired adequate delivery of blood cardioplegic solution, but did not find any group differences. However, to what extent postischemic vasoconstriction contributed to depressed function or other pathophysiologic mechanisms is not clear.

Dr. Richard M. Engelman (Springfield, Mass.). The authors have previously shown that L-arginine benefits the heart when administered in the cardioplegic solution. Have they attempted to see whether L-arginine reverses the effect that was found with $N^{\omega}$-nitro-L-arginine methyl ester when it was just given during reperfusion?

Dr. Sato. No, we did not try to reverse the effects of $N^{\omega}$-nitro-L-arginine methyl ester with L-arginine in this experiment. However, we have done this in other experiments, which have shown that neutrophil accumulation, neutrophil-mediated damage, and increased adherence to endothelium observed with $N^{\omega}$-nitro-L-arginine methyl ester can be reversed by L-arginine, thereby substantiating the NO synthase pathway. 